\title{
Quadratically consistent integration schemes for the element-free Galerkin method
}

\author{
Q. Duan ${ }^{1}$, X. $\mathrm{Li}^{1}, \mathrm{H}$. Zhang $^{1} \& \mathrm{~T}$. Belytschko ${ }^{2}$ \\ ${ }^{1}$ The State Key Laboratory of Structural Analysis for Industrial \\ Equipment, Dalian University of Technology, P. R. China \\ ${ }^{2}$ Department of Mechanical Engineering, Northwestern University, USA
}

\begin{abstract}
This paper presents two efficient integration schemes for the element-free Galerkin (EFG) method. They are able to let EFG with quadratic basis pass the quadratic patch test exactly in a numerical sense. The proposed two schemes, respectively, use three and one quadrature points in each background triangle cell and the derivatives of the nodal shape functions at these quadrature points are corrected by the introduced discrete divergence consistency (DDC) condition. Numerical results of benchmark examples demonstrate the improved accuracy, convergence, efficiency and stability by the proposed schemes.
\end{abstract}

\section{Introduction}

Meshfree Galerkin methods, such as the element-free Galerkin (EFG) method, possess several attractive merits such as the super convergence, the smoothness of the approximation functions and the ease to construct high order approximations, etc. However, accurate integration of the Galerkin weak form in meshfree methods is more difficult than in the finite element method due to the non-polynomial character of meshfree approximants as well as the misalignment between the nodal supports and the integration cells [1]. To ensure a stable solution, higher order Gauss integration is commonly employed in meshfree Galerkin methods, e.g. $4 \times 4$ Gauss quadrature for background quadrilaterals is suggested in [2]. This kind of methods is very costly and, more importantly, it fails to exactly pass the patch tests.

Strategies to accelerate meshfree computations can be found in the nodal integration $[3,4]$, the stress-point integration $[5,6]$ and the support integration 
methods [7, 8]. So far, the most successful and popular method for efficient integration is probably the stabilized conforming nodal integration (SCNI) method proposed by Chen et al. [9]. They showed that by performing a strain smoothing, the linear patch test condition can be exactly satisfied and therefore SCNI can provide even better accuracy than Gauss integration which cannot pass linear patch test exactly. However, the smoothed strain evaluated at the center of each background integration cell can only reproduce a constant strain field in the cell. Consequently, SCNI can only provide accuracies and convergence comparable to linear finite elements even if a quadratic approximation is employed.

This paper presents two integration schemes for meshfree Galerkin methods with quadratic approximations which can reproduce linear strain fields in background integration cells. Quadratic patch test can be exactly passed by the proposed schemes. Consequently, convergence and accuracy are restored to theoretical predictions for quadratic approximations. In addition, efficiency is also greatly improved since the proposed schemes use, respectively, only one and three quadrature points in each background triangle cell.

\section{Consistency framework for nodal derivatives}

First, a framework for the consistency of nodal derivatives is developed, under which the two proposed schemes can be straightforwardly developed. Traditionally, the consistency requirement for the nodal derivatives in meshfree Galerkin methods is only the differentiation of the approximation consistency (DAC) which can be written in a compact form as

$$
\mathbf{p}_{, i}(\mathbf{x})=\sum_{I} \mathbf{p}\left(\mathbf{X}_{I}\right) N_{I, i}(\mathbf{x})
$$

where $\mathbf{p}(\mathbf{x})$ is the vector of base functions and $N_{I}(\mathbf{x})$ are the nodal meshfree shape functions. Note that the DAC is a consistency requirement only among nodal derivatives $N_{I, i}(\mathbf{x})$. The consistency requirement between the smooth function $N_{I}(\mathbf{x})$ and its derivatives $N_{I, i}(\mathbf{x})$ imposed by the divergence theorem should also be satisfied. This requirement can be written as

$$
\int_{\Omega_{S}} N_{I, i}(\mathbf{x}) \mathbf{q}(\mathbf{x}) \mathrm{d} \Omega=\int_{\Gamma_{S}} N_{I}(\mathbf{x}) \mathbf{q}(\mathbf{x}) n_{i} \mathrm{~d} \Gamma-\int_{\Omega_{S}} N_{I}(\mathbf{x}) \mathbf{q}_{, i}(\mathbf{x}) \mathrm{d} \Omega
$$

with $\mathbf{q}(\mathbf{x})=\mathbf{p}_{, x}(\mathbf{x}) \cup \mathbf{p}_{, y}(\mathbf{x})$ for two dimensional case. Especially, for quadratic base $\mathbf{p}(\mathbf{x})=\left[\begin{array}{llllll}1 & x & y & x^{2} & x y & y^{2}\end{array}\right]^{\mathrm{T}}$ considered in this paper, Eq. (2) can be expanded as

$$
\int_{\Omega_{S}} N_{I, x}(\mathbf{x}) \mathrm{d} \Omega=\int_{\Gamma_{S}} N_{I}(\mathbf{x}) n_{x} \mathrm{~d} \Gamma
$$




$$
\begin{gathered}
\int_{\Omega_{S}} N_{I, x}(\mathbf{x}) x \mathrm{~d} \Omega=\int_{\Gamma_{S}} N_{I}(\mathbf{x}) x n_{x} \mathrm{~d} \Gamma-\int_{\Omega_{S}} N_{I}(\mathbf{x}) \mathrm{d} \Omega \\
\int_{\Omega_{S}} N_{I, x}(\mathbf{x}) y \mathrm{~d} \Omega=\int_{\Gamma_{S}} N_{I}(\mathbf{x}) y n_{x} \mathrm{~d} \Gamma
\end{gathered}
$$

for the derivatives with respect to $x$. Note that this requirement should be satisfied in discrete sense, i.e. the domain and boundary integrations in Eq. (2) should be performed numerically. Therefore, it is called the discrete divergence consistency (DDC) for the nodal derivatives. The proposed consistency framework includes both the DAC and the DDC, i.e. both conditions should be satisfied by nodal derivatives.

\section{Quadratically consistent 3-point (QC3) integration scheme}

Under the proposed framework, we can design efficient integration schemes if we compute the nodal derivatives at quadrature points by the DDC. First, we design a 3-point integration scheme as shown in Figure 1. The dark dots are the nodes which are used to construct the meshfree approximation. The symbol orange triangles denote the vertices of the background triangle mesh for the purpose of domain integration. In each background triangle element (cell), we use three quadrature points denoted by red crosses. The blue stars on the edges of the background triangles are the one dimensional Gauss points for boundary integrations.

Obviously, the three equations (3)-(5) can be used to determine the derivatives at the three quadrature points, i.e. the derivatives can be directly computed by the DDC. After the integration is performed numerically, the discrete version of Eqs (3)-(5), i.e. the DDC, can be written as

$$
\mathbf{W} \mathbf{d}_{x}=\mathbf{f}_{x}
$$

where

$$
\begin{aligned}
& \mathbf{W}=\left[\begin{array}{ccc}
W_{1} & W_{2} & W_{3} \\
W_{1} x_{1} & W_{2} x_{2} & W_{3} x_{3} \\
W_{1} y_{1} & W_{2} y_{2} & W_{3} y_{3}
\end{array}\right] \quad \mathbf{d}_{x}=\left\{\begin{array}{c}
N_{I, x}\left(\mathbf{x}_{1}\right) \\
N_{I, x}\left(\mathbf{x}_{2}\right) \\
N_{I, x}\left(\mathbf{x}_{3}\right)
\end{array}\right\} \\
& \sum_{L=1}^{3} \sum_{G=1}^{2} N_{I}\left(\mathbf{x}_{G}\right) n_{x}^{L} w_{G} \\
& \mathbf{f}_{x}=\left\{\begin{array}{c}
\left.\sum_{L=1}^{3} \sum_{G=1}^{2} N_{I}\left(\mathbf{x}_{G}\right) x_{G} n_{x}^{L} w_{G}-\sum_{H=1}^{3} W_{H} N_{I}\left(\mathbf{x}_{H}\right)\right\} \\
\sum_{L=1}^{3} \sum_{G=1}^{2} N_{I}\left(\mathbf{x}_{G}\right) y_{G} n_{x}^{L} w_{G}
\end{array}\right\}
\end{aligned}
$$

Note that the nodal derivatives computed by Eq. (6) can satisfy both the quadratic DAC and the quadratic DDC (see [10] for details). Therefore they are quadratically consistent and can make the method pass the quadratic patch test exactly in a numerical sense which is shown in Table 1. 


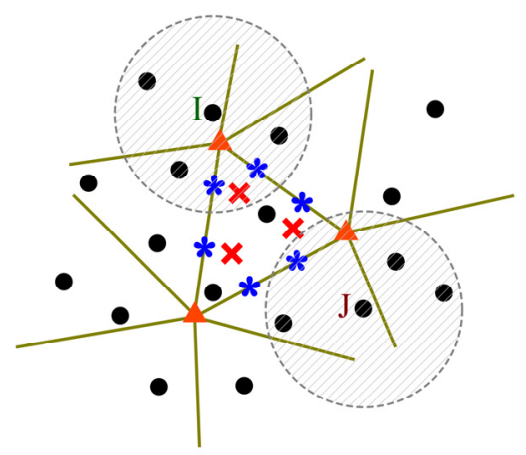

Figure 1: QC3 integration scheme.

Table 1: $\quad$ QC3 scheme: results of quadratic patch test.

\begin{tabular}{cccccccc}
\hline & QC3 & LC1 & SH1 & SH3 & SH7 & SH16 & LFEM \\
\hline$E^{\text {disp }}$ & $\mathbf{0 . 8 3 E - ~}$ & $0.13 \mathrm{E}-$ & $0.40 \mathrm{E}-$ & $0.24 \mathrm{E}-$ & $0.51 \mathrm{E}-$ & $0.25 \mathrm{E}-$ & $0.19 \mathrm{E}-02$ \\
$E^{\mathrm{eng}}$ & $\mathbf{0 . 3 6 E}-$ & $0.99 \mathrm{E}-$ & $0.20 \mathrm{E}-$ & $0.11 \mathrm{E}-$ & $0.29 \mathrm{E}-$ & $0.11 \mathrm{E}-$ & $0.12 \mathrm{E}+00$ \\
\hline
\end{tabular}

Figures 2-4 show the numerical results of the benchmark example plate with a hole. Obviously, the proposed QC3 achieves the best numerical performance in terms of accuracy, convergence, efficiency and stability. Here, LC1 corresponds to SCNI and SH16 stands for standard EFG method with 16 quadrature points in each background triangle cell.

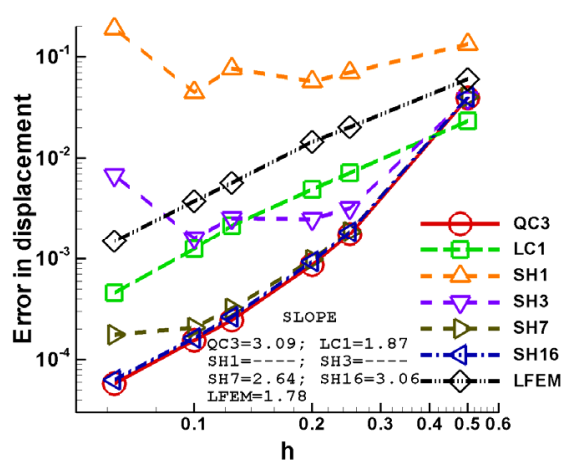

(a)

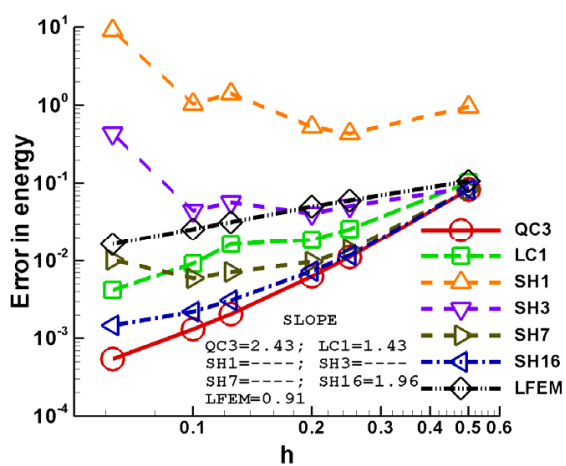

(b)

Figure 2: Convergence for the plate with a hole problem: (a) displacement; (b) energy. 


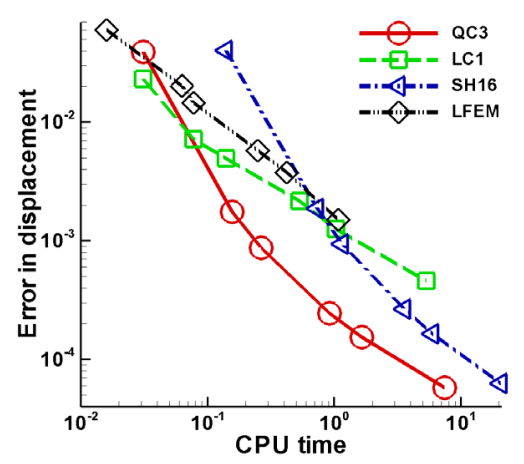

(a)

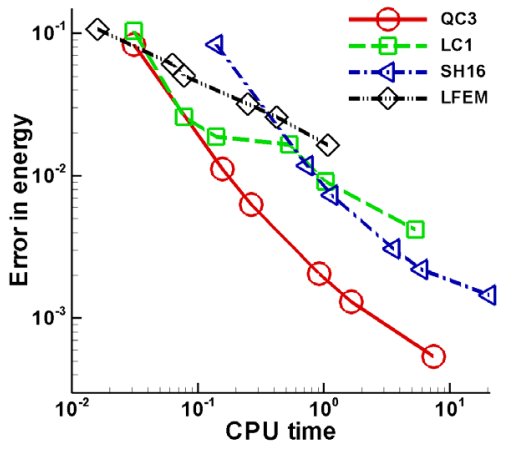

(b)

Figure 3: Computational efficiency for the plate with a hole problem in terms of: (a) displacement; (b) energy.

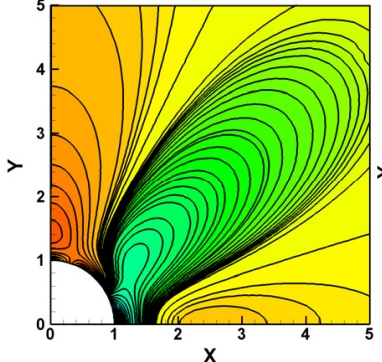

(a)

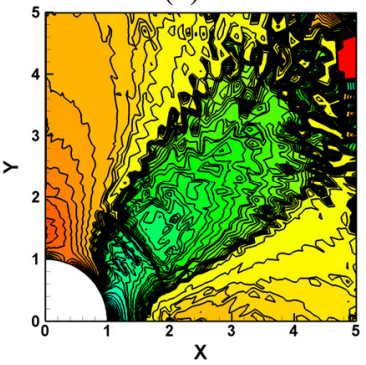

(d)

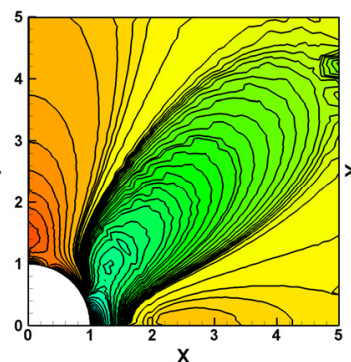

(b)

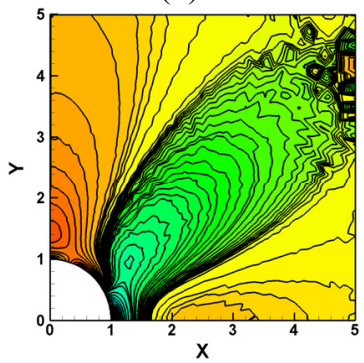

(e)

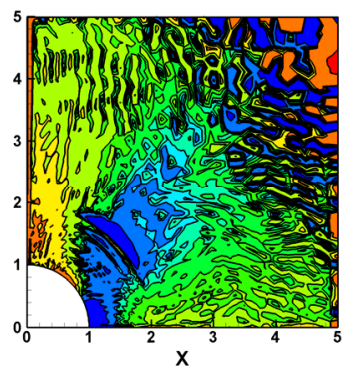

(c)

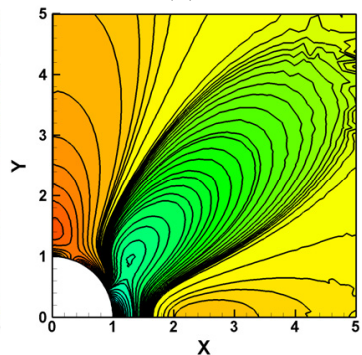

(f)

Figure 4: $\quad \sigma_{y y}$ stress fields of the plate with a hole problem by EFG with the integration schemes of: (a) QC3; (b) LC1; (c) $\mathrm{SH}$; (d) $\mathrm{SH}$; (e) $\mathrm{SH} 7$; (f) SH16. 


\section{Quadratically consistent 1-point (QC1) integration scheme}

Note that, in the proposed QC3 scheme, three quadrature points can exactly reproduce a linear strain field for each background integration cell. In fact, this also can be achieved by only one quadrature point each cell with the help of high order derivatives which can be naturally introduced by the Taylor's expansion. This is the basic idea for developing quadratically consistent 1-point (QC1) scheme based on QC3 (see [11] for details).

Table 2 and Figures 5-7 show the numerical results of the proposed QC1 scheme in quadratic patch test and a manufactured problem. Clearly, QC1 passed the quadratic patch test exactly in a numerical sense and achieved very good numerical performance.

Table 2: $\quad$ QC1 scheme: results of quadratic patch test.

\begin{tabular}{ccccc}
\hline & Direct1 & TEBS1 & LC1 & QC1 \\
\hline$E^{\text {disp }}$ & $0.30 \mathrm{E}-02$ & $0.56 \mathrm{E}-03$ & $0.24 \mathrm{E}-02$ & $0.37 \mathrm{E}-12$ \\
$E^{\text {eng }}$ & $0.81 \mathrm{E}-02$ & $0.16 \mathrm{E}-02$ & $0.63 \mathrm{E}-02$ & $0.10 \mathrm{E}-11$ \\
\hline
\end{tabular}

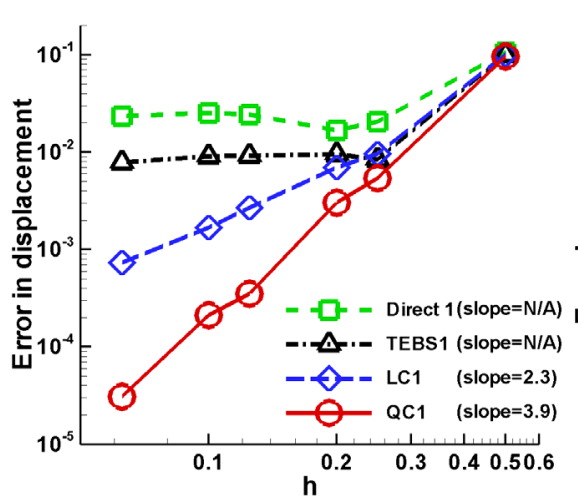

(a)

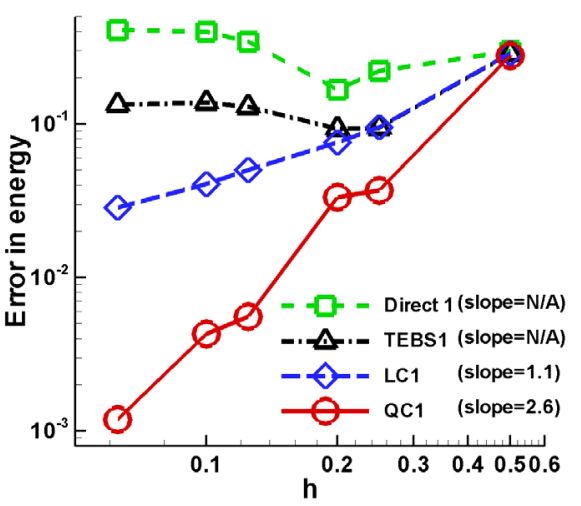

(b)

Figure 5: Convergence of the manufactured problem: (a) displacement; (b) energy. 


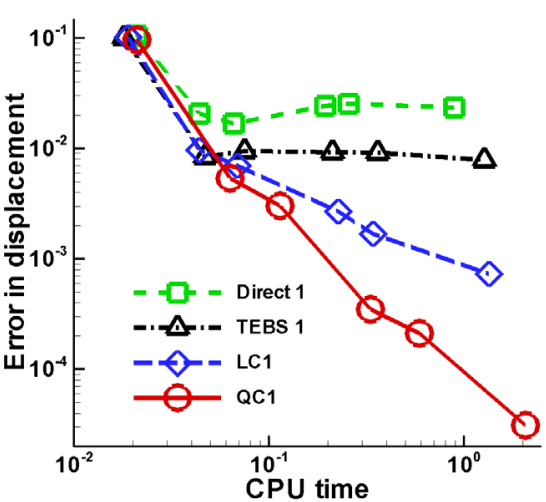

(a)

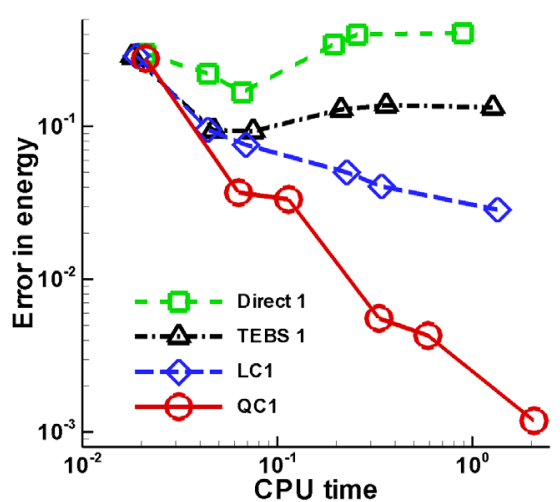

(b)

Figure 6: Computational efficiency of the manufactured problem: (a) displacement; (b) energy.

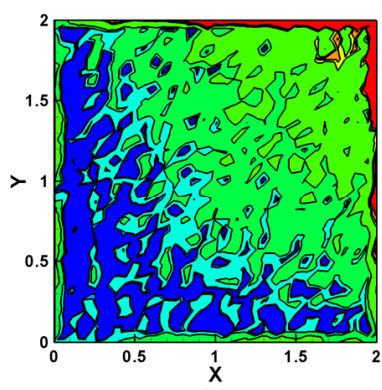

(a)

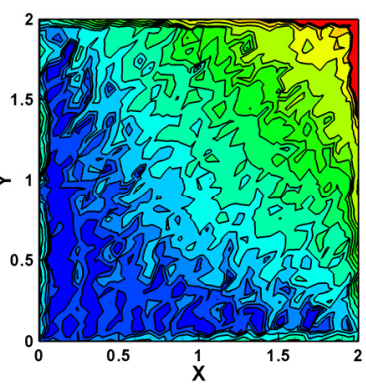

(b)

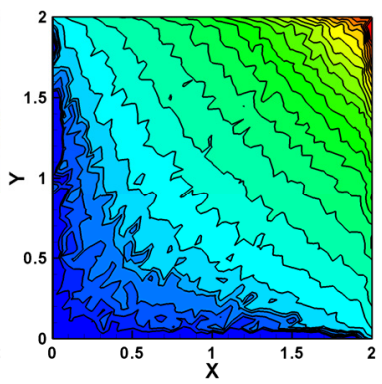

(c)

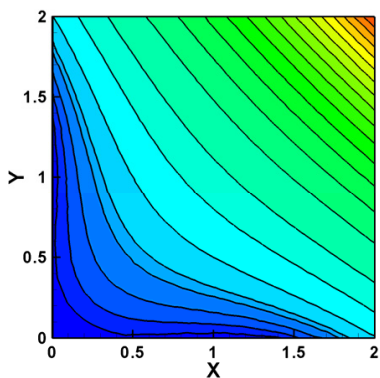

(d)

Figure 7: $\quad \sigma_{x x}$ stress fields of the manufactured problem by EFG with the integration schemes of: (a) Direct 1; (b) TEBS1; (c) LC1; (d) QC1.

\section{Conclusion}

A framework for the consistency of nodal derivatives including both the DAC and the DDC is proposed, under which two integration schemes, i.e. QC3 and 
QC1, are developed. Numerical results show that both schemes exactly passed quadratic patch test and achieved promising numerical performance.

\section{Acknowledgements}

The authors are pleased to acknowledge the support of this work by the National Natural Science Foundation of China through contract/grant numbers 11102036, 11072046 and 11232003, the National Key Basic Research and Development Program (973 Program, No. 2010CB731502) and the Fundamental Research Funds for the Central Universities through grant number DUT12LK08.

\section{References}

[1] Dolbow J, Belytschko T. Numerical integration of the Galerkin weak form in meshfree methods. Computational Mechanics 1999; 23: 219-230.

[2] Belytschko T, Lu YY, Gu L. Element-free Galerkin methods. International Journal for Numerical Methods in Engineering 1994; 37: 229-256.

[3] Beissel S, Belytschko T. Nodal integration of the element-free Galerkin method. Computer Methods in Applied Mechanics and Engineering 1996; 139: 49-74.

[4] Liu GR, Zhang GY, Wang YY, Zhong ZH, Li GY, Han X. A nodal integration technique for meshfree radial point interpolation method (NIRPIM). International Journal of Solids and Structures 2007; 44: 38403860 .

[5] Fries TP, Belytschko T. Convergence and stabilization of stress-point integration in mesh-free and particle methods. International Journal for Numerical Methods in Engineering 2008; 74: 1067-1087.

[6] Duan QL, Belytschko T. Gradient and dilatational stabilizations for stresspoint integration in the element-free Galerkin method. International Journal for Numerical Methods in Engineering 2009; 77: 776-798.

[7] Kwon KC, Park SH, Youn SK. The support integration scheme in the leastsquares mesh-free method. Finite Elements in Analysis and Design 2006; 43: $127-144$.

[8] Liu Y, Belytschko T. A new support integration scheme for the weakform in mesh-free methods. International Journal for Numerical Methods in Engineering 2010; 82: 699-715.

[9] Chen JS, Wu CT, Yoon S, You Y. A stabilized conforming nodal integration for Galerkin mesh-free methods. International Journal for Numerical Methods in Engineering 2001; 50: 435-466.

[10] Duan QL, Li XK, Zhang HW, Belytschko T. Second-order accurate derivatives and integration schemes for meshfree methods. International Journal for Numerical Methods in Engineering 2012; 92: 399-424.

[11] Duan QL, Li XK, Zhang HW, et al. Quadratically consistent one-point (QC1) quadrature for meshfree Galerkin methods. Computer Methods in Applied Mechanics and Engineering 2012; 245-246: 256-272. 